\title{
The Number of Spanning Trees in Graphs with a Given Degree Sequence
}

\author{
A. V. Kostochka* \\ Institute of Mathematics, Siberian Branch of the Russian Academy of Sciences, \\ 630090 Novosibirsk, Russia
}

Dedicated to Professor Paul Erdös on the occasion of his 80th birthday

\section{ABSTRACT}

Alon's [1] idea is slightly refined to prove that for each connected graph $G$ with degree sequence $1<k=d_{1} \leq d_{2} \leq \cdots \leq d_{n}$ the number $C(G)$ of spanning trees of $G$ satisfies the inequality

$$
d(G) k^{-n o(\log k / k)} \leq C(G) \leq d(G) /(n-1),
$$

where $d(G)=\left(\Pi_{i=1}^{n} d_{i}\right)$. An almost exact lower bound for $C(G)$ for 3-regular $G$ on $n$ vertices is also given. (C) 1994 John Wiley \& Sons, Inc.

\section{INTRODUCTION}

All graphs considered here are simple connected graphs. For a graph $G$ on $n$ vertices let $C(G)$ denote the number of spanning trees of $G$ and $c(G)=(C(G))^{1 / n}$. It is known (see $[1,2])$ that for each $k$-regular graph $G(k>1)$,

$$
c(G)<k \text {. }
$$

Alon [1] studied $c(k)=\lim _{n \rightarrow \infty} \inf \{c(G) \mid G$ is $k$-regular on $n$ vertices $\}$.

Theorem A [1]. For each $k$-regular graph $G$,

* This work was partly supported by the Grant 93-011-1486 of the Russian Foundation of Fundamental Research.

Random Structures and Algorithms, Vol. 6, Nos. 2 and 3 (1995)

(C) 1995 John Wiley \& Sons, Inc. CCC 1042-9832/95/030269-06 


$$
c(G) \geq k^{1-O\left((\log \log k / \log k)^{2}\right)} .
$$

Therefore, $c(k) \geq k^{1-O\left((\log \log k / \log k)^{2}\right)}$.

Theorem B [1]. For each $k>2, \sqrt{ } 2 \leq c(k)<\left((k+1)^{k-2}(k-1)\right)^{1 /(k+1)}=$ $k^{1-\Theta(1 / k)}$. In particular, $2^{1 / 2} \leq c(3) \leq 2^{3 / 4}$.

The result of Theorem $A$ together with (1) shows that the structure of a $k$-regular graph $G$ unexpectedly does not affect too strongly the quantity $C(G)$. For example, if $k$ is large enough, then we have $C(G)<C(H)$ for any $k$-regular $G$ and $1.001 k$-regular $H$ on the same number of vertices. In this article we will follow the lines of Alon's proof to sharpen and generalize Theorem A. The main result is

Theorem 1. Let $G$ be a connected graph with degree sequence $1<k=d_{1} \leq d_{2} \leq$ $\cdots \leq d_{n}$. Denote $d(G)=\left(\Pi_{i=1}^{n} d_{i}\right)$. Then $d(G) k^{-n O(\log k / k)} \leq C(G) \leq d(G) /(n-$ 1). Therefore, $k^{1-o(\log k / k)} \leq c(k) \leq k^{1-\Theta(1 / k)}$.

This means that the dependence of $C(G)$ on the structure of $G$ for graphs with large minimal degree is rather weak. In [1] Alon asked about exact values of $c(k)$. We find here $c(3)=2^{3 / 4}$ by proving

Theorem 2. Let $G$ be a graph whose vertex degrees are from $\{2,3\}$, having $m$ vertices of degree 3 . Then

$$
C(G) \geq 2^{3(m+2) / 4},
$$

provided $G \neq K_{4}$.

\section{A LOWER BOUND FOR LARGE $k$}

Let $G$ be a (connected) graph with degree sequence $k=d_{1} \leq d_{2} \leq \cdots \leq d_{n}$ of vertices $v_{1}, v_{2}, \ldots, v_{n}$. Following Alon [1], for each $i \in\{1, \ldots, n\}$ choose, randomly (with a uniform distribution on the $d_{i}$ vertices adjacent to $v_{i}$ ) and independently, a vertex $\Gamma\left(v_{i}\right)$ adjacent to $v_{i}$ and orient the edge $\left(v_{i}, \Gamma\left(v_{i}\right)\right)$ from $v_{i}$ to $\Gamma\left(v_{i}\right)$. The number of components in the resulting oriented subgraph $H$ of $G$ is equal to the number of oriented cycles in $H$ (oriented cycles of length 2 are possible). Note that the number of possible resulting oriented subgraphs $H$ is $d(G)$. Every spanning tree $T$ of $G$ will be represented among these $H$ exactly $n-1$ times (with cycles of length 2 using any one edge of $T$ ). Thus, $C(G) \leq d(G)$ / $(n-1)$.

Lemma 1. For each $i \in\{1, \ldots, n\}$ and any integer $t>1$ the probability that $v_{i}$ belongs to an oriented cycle in $H$ of length $t$ is at most $1 / k$.

Proof. Since $\Gamma\left(v_{i}\right)$ are chosen independently, we can consider the events consecutively. Let $w_{0}=v_{i}$ and, for $j=1,2, \ldots, t-1, w_{j}=\Gamma\left(w_{j-1}\right)$. If not all the vertices $w_{0}, w_{1}, \ldots, w_{t-1}$ are distinct or $\left(w_{0}, w_{t-1}\right)$ is not an edge in $G$, then $v_{i}$ 
does not belong to an oriented cycle in $H$ of length $t$. Otherwise, the probability that $v_{i}=\Gamma\left(w_{t-1}\right)$ is $1 / d\left(w_{t-1}\right) \leq 1 / k$.

Lemma 2. For any integer $t>1$ the expectation of the number of components of $H$ with oriented cycles of length at most $t$ is no more than $(n / k) \ln t$.

Proof. By Lemma 1, the expected number of vertices on oriented cycles in $H$ of length $j$ is at most $n / k$. Hence the expected number of such cycles is at most $n / k j$. Thus, the desired expectation does not exceed $(n / k)(1 / 2+1 / 3+\cdots+1 / t)$.

To prove Theorem 1, it is enough now just to repeat the second half of Alon's proof [1] of Theorem A, keeping in mind Lemma 2. Thus, we only outline the arguments. Let $\mathscr{H}$ be the family of those oriented subgraphs $H$ of $G$ having at most $(2 n / k) \ln k$ components of size $k$ or less. Then each $H \in \mathscr{H}$ has less than $(3 n / k) \ln k$ components. By Lemma $2,|\mathscr{H}| \geq d(G) / 2$. With each $H \in \mathscr{H}$ we associate a forest $F_{H}$ by deleting an arbitrary edge from the unique cycle of each component. Then it can be seen that any such forest $F$ can be obtained from at most $k^{(6 n / k) \ln k}$ distinct $H \in \mathscr{H}$. Each $F_{H}$ is contained in a spanning tree of $G$ and any spanning tree of $G$ contains at most

$$
\sum_{i=0}^{(3 n / k) \ln k}\left(\begin{array}{c}
n-1 \\
i
\end{array}\right)=k^{n O(\log k / k)}
$$

forests with less than $(3 n / k) \ln k$ components. This completes the proof of Theorem 1.

\section{ON GRAPHS WITH MAXIMAL DEGREE 3}

Throughout the section for a graph $H$ we will denote by $m(H)$ the number of vertices of degree 3 in $H$ and $f(H)=2^{3(m(H)+2) / 4}$.

Let us count $C(G)$ for several $G$. Denote $H_{1}=K_{4}, H_{2}=K_{4} \backslash e, H_{3}=K_{3,3}$, $H_{4}$-3-prism; to obtain $H_{5}$, we subdivide an edge of $K_{4}$ by a vertex. It is an easy exercise to see that $C\left(H_{1}\right)=16, C\left(H_{2}\right)=8, C\left(H_{3}\right)=81, C\left(H_{4}\right)=75, C\left(H_{5}\right)=24$. Thus, for $\mathrm{H}_{2}-\mathrm{H}_{5}$, Theorem 2 is true.

Proof of Theorem 2. Suppose that $G=(V, E)$ is a minimum (on the number of edges) graph which is not $K_{4}$ such that $C(G)<f(G)$. Evidently, $|E|>3$.

Claim 1. G has no cut-edge.

Proof. If $G$ has a cut-edge, then for some $s$ there is a path $P=\left(v_{1}, \ldots, v_{s}\right)$ such that $d\left(v_{1}\right)=d\left(v_{s}\right)=3, d\left(v_{2}\right)=\cdots=d\left(v_{s-1}\right)=2$ and all the edges of $P$ are cutedges. Let $G_{1}$ and $G_{2}$ be the components of the graph obtained from $G$ by deleting the edges and the interior vertices of $P$. Both $G_{1}$ and $G_{2}$ have a vertex of degree 2 (namely, $v_{1}$ and $v_{s}$ ) and hence do not coincide with $K_{4}$. Note that $m\left(G_{1}\right)+m\left(G_{2}\right)=m(G)-2$. By the minimality of $G, C\left(G_{1}\right) C\left(G_{2}\right) \geq f\left(G_{1}\right) f\left(G_{2}\right)=$ $f(G)$. But $C(G)=C\left(G_{1}\right) C\left(G_{2}\right)$, a contradiction. 
Claim 2. $G$ is 3-regular.

Proof. Suppose that $G$ contains a vertex $v$ with $N_{G}(v)=\{x, y\}$.

Case 1. $(x, y) \notin E$. Let $G_{1}=(G \backslash v) \cup\{(x, y)\}$. If $G_{1}=K_{4}$, then $G=H_{5}$, a contradiction. Otherwise by the minimality of $G, C\left(G_{1}\right) \geq f\left(G_{1}\right)=f(G)$. But $C(G) \geq C\left(G_{1}\right)$.

Case 2. $(x, y) \in E$. If $d(y)=2$, then either $G=K_{3}$ and we are done, or the third edge incident with $x$ is a cut-edge, which contradicts Claim 1. So, we can suppose that $N_{G}(x)=\{v, y, u\}, N_{G}(y)=\{v, x, w\}$. If $u=w$, then again either $G=K_{4} \backslash e=H_{2}$ and we are done, or the third edge incident with $w$ is a cut-edge. Thus, we can assume $u \neq w$. Let $G_{1}$ be obtained from $G$ by contracting $x, y$, and $v$ into a new vortex $z$ (of degree 2). By the minimality of $G, C\left(G_{1}\right) \geq f\left(G_{1}\right)=$ $2^{-3 / 2} f(G)$. But each spanning tree of $G_{1}$ can be extended to a spanning tree of $G$ by three ways [by adding any two edges of the triangle $(x, v, y)]$. Hence $C(G) \geq$ $3 C\left(G_{1}\right) \geq 3 f\left(G_{1}\right)>f(G)$.

Claim 3. G contains no subgraph $K_{4} \backslash$ e.

Proof. Suppose that $G$ contains a subgraph $K_{4} \backslash$ le (see Fig. 1). Since $G$ has no cut-edge, $w \neq v$. Consider $G_{1}=G \backslash\{x, y, z, u\}$. By the minimality of $G[d(w)=2$ in $\left.G_{1}\right], C\left(G_{1}\right) \geq f\left(G_{1}\right)=2^{-4.5} f(G)$. Because of $C\left(K_{4} \backslash e\right)=8$, each spanning tree $T_{1}$ of $G_{1}$ can be extended to a spanning tree $T$ of $G$ by:

(a) 8 ways such that $T$ contains $(x, w)$ and does not contain $(u, v)$;

(b) 8 ways such that $T$ contains $(u, v)$ and does not contain $(x, w)$;

(c) 8 ways such that $T$ contains both $(u, v)$ and $(x, w)$.

Hence $C(G) \geq 24 C\left(G_{1}\right)>f(G)$.

Claim 4. G contains no triangles.

Proof. Suppose that $G$ contains a triangle $(x, y, z)$ and $\{(x, u),(y, v),(z, w)\} \subset$ $E$. Due to Claim 3 , the vertices $u, v$, and $w$ are distinct. Let $G_{1}$ be obtained from $G$ by contracting $x, y$, and $z$ into a new vertex $t$. If $G_{1}=K_{4}$, then $G=H_{4}$, a contradiction. Otherwise, by the minimality of $G, C\left(G_{1}\right) \geq f\left(G_{1}\right)=2^{-3 / 2} f(G)$. But

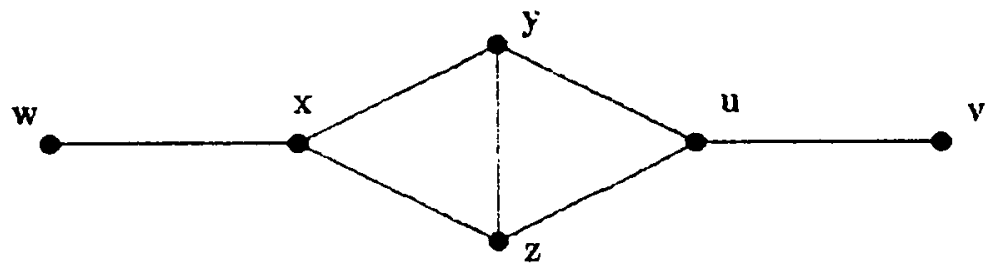

Fig. 1. 
each spanning tree of $G_{1}$ can be extended to a spanning tree of $G$ by three ways [by adding any two edges of the triangle $(x, y, z)$ ]. Hence $C(G) \geq 3 C\left(G_{1}\right) \geq$ $3 f\left(G_{1}\right)>f(G)$.

Now, consider the neighborhood of an arbitrary edge $(u, v)$ of $G$ [see Fig. 2(a)]. Consider $G_{1}=(G \backslash\{v, u\}) \cup\{(x, y),(z, w)\}$ [see Fig. 2(b)]. If $G_{1}=K_{4}$, then $G=H_{3}$, a contradiction. Otherwise, by the minimality of $G, C\left(G_{1}\right) \geq f\left(G_{1}\right)=$ $2^{-1.5} f(G)$. For each spanning tree $T_{1}$ of $G_{1}$, we will point out three spanning trees of $G$ containing $T_{1}$ such that any spanning tree $T$ of $G$ will appear at most once.

Case 1. $E\left(T_{1}\right) \cap\{(x, y),(z, w)\}=\emptyset$. We extend $T_{1}$ by four ways adding an edge from $\{(x, u),(y, u)\}$ and an edge from $\{(z, v),(w, v)\}$.

Case 2. $(x, y) \in E\left(T_{1}\right),(z, w) \notin E\left(T_{1}\right)$. We add to $E\left(T_{1}\right)$ the edges $(x, u)$, $(y, u)$, and an edge incident to $u$ (three ways).

Case 3. $(x, y) \notin E\left(T_{1}\right),(z, w) \in E\left(T_{1}\right)$. We do symmetrically to the Case 2 .

Case 4. $E\left(T_{1}\right) \supset\{(x, y),(z, w)\}$. Then in $T_{1} \backslash\{(x, y),(z, w)\}$ exactly one pair of elements of $\{x, y, z, w\}$ is connected by a path, and this pair is neither $\{x, y\}$ nor $\{z, w\}$. W.1.o.g. we suppose that this pair is $\{z, x\}$. Then we add to $E\left(T_{1}\right)$ the sets (a) $\{(x, u),(y, u),(z, v),(w, v)\},(\mathrm{b})\{(v, u),(y, u),(z, v),(w, v)\}$, and (c) $\{(v, u)$, $(y, u),(x, u),(w, v)\}$.

To construct the examples for which equality in (2) holds, consider an arbitrary tree $T$ with the maximal degree 3 . Now, attach to every end-vertex $v$ of $T$ a copy of $K_{4} \downarrow e$ so that the degree of $v$ becomes 3, and for some subset $A$ of $E(T)$ replace each edge $(v, w)$ by a copy of the graph on Figure 1 (with $v$ and $w$ as on Figure 1). Denote the resulting graph by $G(T, A)$. Recall that the number of end-vertices of $T$ is equal to $m(T)+2$. Since $C\left(K_{4} \backslash e\right)=8, m(G(T, A))=4(m(T)+2+|A|)-2$ and each cut-edge belongs to any spanning tree, we have $C(G(T, A))=8^{m(T)+2+|A|}$ $=8^{(m(G(T, A))+2) / 4}$. It can be proved that any graph with equality in $(2)$ can be obtained from some $G(T, A)$ by replacing several paths by edges.

As to 3-regular graphs, consider a path $P_{t}=\left(v_{1}, \ldots, v_{2 t}\right)$. Attach to each end of $P_{t}$ a copy of $H_{5}$ described above and replace for $i=1, \ldots, t-1$ the edge $\left(v_{2 i}, v_{2 i+1}\right)$ by a copy of $K_{4}$ le so that the resulting graph $G(t)$ is 3-regular. We know that $C\left(H_{5}\right)=24$, and $m(G(t))=|V(G(t))|=6+4 t=4(t+2)-2$. Hence, $C(G(t))=24^{2} 8^{t-1}=(9 / 8) 8^{(m(G(t))+2) / 4}=(9 / 8) f(G(t))$. Thus, the bound here is also

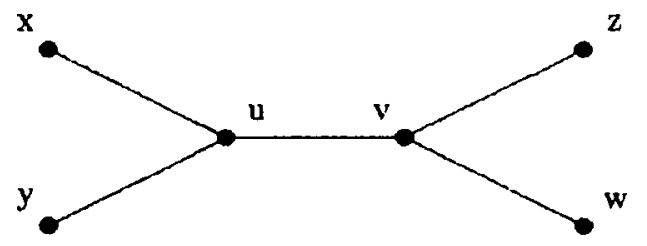

a)

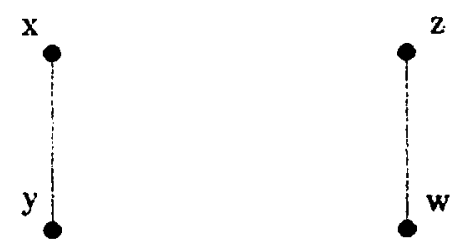

b)

Fig. 2. 
close to the truth. More careful consideration can show that $(9 / 8) f(G)$ is the lower bound of $C(G)$ for 3-regular graphs $G$ except for $K_{4}$.

\section{ACKNOWLEDGMENT}

The author thanks N. Alon for interesting and useful discussions and other help.

\section{REFERENCES}

[1] N. Alon, The number of spanning trees in regular graphs, Random Struct. Alg., 1 175-181 (1990).

[2] B. D. McKay, Spanning trees in regular graphs, Eur. J. Combinat., 4 149-160 (1983).

Received November 2, 1993

Accepted April 14, 1994 